\title{
Immune system and quality of life following aerobic exercise versus resistance exercise training among Alzheimer's
}

\author{
Fadwah M Al-Sharif* \\ Department of Medical Laboratory Technology, Faculty of Applied Medical Sciences, King \\ Abdulaziz University, Jeddah, Saudi Arabia
}

\section{Abstract}

Background: Globally, Alzheimer's disease $(A D)$ affects millions of elderly individuals are affected with $A D$ who suffer from decline in cognitive ability. However, immune system dysfunction has a role in $A D$ pathogenesis. However, pharmacological therapeutic intervention for caring of ADis not available. Therefore there is a need to develop novel therapeutic modalities for AD individual care.

Objective: The objective of the this trial was to detect immune system and quality of life $(Q O L)$ response following aerobic versus resisted exercise training among $A D$ subjects.

Methods: Fifty older with $A D$ disease the range of age ranged was 61 to 73 years enrolled in the current study. However, smoking, liver, chest, renal, metabolic and cardiac dysfunction considered as exclusion criteria. Participants were randomly enrolled into group (A) who applied aerobic exercise intervention, while group $(B)$ applied resisted exercise intervention for period of six months.

Results: The SF-36 which measure QOL along with in the immunological parameters (CD3 count, CD4 count, CD8 count and CD4/CD8 ratio) showed significant improvement following aerobic and resisted exercise. However, comparing between both groups showed significant differences with greater significant improvement in all measured parameters following aerobic exercise training $(p<0.05)$.

Conclusion: Aerobic exercise is the most appropriate exercise to improve immune system and quality of life among elderly Alzheimer's.

\section{Introduction}

Alzheimer's disease (AD) characterized with deteriorated both function, cognition and neuronal loss [1]. Currently, there is about 24 million $\mathrm{AD}$, which expected to reach about 100 million subjects by 2050 around the world [2,3]. The cost of management of the current $\mathrm{AD}$ population is greater than 220 billion US dollars, which is expected to reach greater than one trillion by 2050 [4]. Both patient and family are adversely affected by $\mathrm{AD}$ as the disease badly deteriorate behavior, cognitive and physical aspects [5].

Evidences proved an association between inflammation of brain tissue and aging changes that drive the pathological changes in $\mathrm{AD}$ [6]. The inflammatory changes induces immunological changes promotes $\mathrm{AD}$ development $[7,8]$.

\section{More Information}

*Address for Correspondence: Dr. Fadwah M Al-Sharif, Department of Medical Laboratory Technology, Faculty of Applied Medical Sciences, King Abdulaziz University, P.O. Box 80324, Jeddah, 21589, Saudi Arabia, Email:drfadwa6@gmail.com

Submitted: 20 April 2020

Approved: 29 April 2020

Published: 30 April 2020

How to cite this article: Al-Sharif FM. Immune system and quality of life following aerobic exercise versus resistance exercise training among Alzheimer's. Arch Asthma Allergy Immunol. 2020; 4: 003-008.

DOI: 10.29328/journal.aaai.1001018

Copyright: (c) 2020 Al-Sharif FM. This is an open access article distributed under the Creative Commons Attribution License, which permits unrestricted use, distribution, and reproduction in any medium, provided the original work is properly cited.

Keywords: Aerobic exercise; Aging; Alzheimer's; Resisted exercises; Immune system; Quality of life.

\section{Check for updates}

OPEN ACCESS
Moreover, the majority of AD individuals suffer from many psychiatric disturbances and depression [9-11]. Therefore there is a need to develop novel therapeutic modalities for AD individual care [12].

Evidences reported positive influence of exercise training on immune system [13]. Therefore, it is usually recommended to apply exercise training to positively influence immune system through modulation of systemic inflammatory status and cognitive dysfunction among AD individuals [14-20]. Limited evidences available regarding differences of influence of aerobic and resisted exercises on elderly with AD. The objective of the this trial was to detect immune system and quality of life(QOL) response following aerobic versus resisted exercise training among AD subjects. 


\section{Patients and methods}

\section{Subjects}

Fifty older with $\mathrm{AD}$ disease the range of age ranged was 61 to 73 years enrolled in the current study. However, smoking, liver, chest, renal, metabolic and cardiac dysfunction considered as exclusion criteria. Participants were randomly enrolled into group (A) who applied aerobic exercise intervention, while group (B) applied resisted exercise intervention for period of six months. A consent form was signed by legal guardian of all participants before sharing in our study which was ethically approved by FAMS Ethical Research Committee, King Abdulaziz University.

\section{Measurements}

A. Flow cytometry analysis: Leukocyte differentiation antigens CD3, CD4 and CD8 were measured using Beckman Coulter, Marseille, France. However, flow cytometry (Cytomics FC 500 and CXP software was used for analysis of samples.

B. Health-related quality of life (SF-36 HRQL): Assessment of quality of life (QOL) was detected using the standard reliable test SF-36 that includes eight subscales: Vitality, Bodily Pain, General Health, Physical Functioning, Social Functioning, Physical Role Functioning, Emotional Role Functioning, and Mental Health [21].

\section{Procedures}

1- Group (A): Twenty-five AD elderly subjects received treadmill aerobic exercise training for six months, the training program started with five minutes warming up, thirty minutes of training on the treadmill with an intensity $60 \%-80 \%$ of individual maximal heart rate and end with five minutes cooling down [22].

2- Group (B): Twenty-five AD elderly subjects received resisted exercise training for six months, the training program started with ten minutes of mobility exercises followed by resisted exercise training on 9 machines of resistance training in order to do resisted training for the main skeletal muscles of the lower limbs, upper limbs and trunk muscles. Training session included 3 sets each set which consisted of 8-12 repetitions with a moment rest in between each 2 set. Training intensity was $60 \%-80 \%$ of one maximum repetition (1-RM) [23].

\section{Results}

Baseline variables of all participants proved that both groups were homogeneous as comparing the both groups regarding baseline and demographic parameters revealed no significant differences (Table 1).

The main findings in the current study showed significant increase in the mean values of SF-36 which measure QOL along with significant improvement in the immunological parameters (CD3 count, CD4 count, CD8 count and CD4/CD8 ratio) following aerobic and resisted exercise training (Tables 2-5). However, comparing between both groups showed significant differences with greater significant improvement in all measured parameters following aerobic exercise training (Tables 6,7).

\section{Discussion}

Globally, millions of elderly individuals are affected with AD who suffer from decline in cognitive ability. However, immune system dysfunction play a principal role in $\mathrm{AD}$ pathogenesis $[24,25]$. Recently, there is no available pharmacological therapeutic intervention for caring of AD. However, exercise training is of positive effects on physical and cognitive function of AD individuals [26,27].

Results of current study proved that after six months, immunological parameters (CD3, CD4 and CD8) significantly increased and CD4/CD8 ratio significantly decreased in group (A) applied aerobic exercises greater than group (B) applied resisted exercises. Cell numbers are expected to decrease due to aging process. This finding is consistent with other studies, while other contradicting studies have made different observations. As our results agreed with many researches proved that aerobic exercise training improved immune system of elderly subjects [28-31]. However, Peeri and colleagues, stated that number of CD4 \& CD8 cells and physical fitness significantly increased following aerobic exercise for six months in healthy elderly males [32]. While, Kapasi, et al. reported that two months of combined aerobic and resisted exercises improved CD8+ cells among elderly individuals [33]. On the other hand, limited evidences that measure influence of immunological response to resisted exercise are available, the majority of these studies reported that 2-3 months of resisted exercise programs had minimal influence of immune system and inflammatory cytokines [34-38]. While, many studies did not show significant immune function improvement following progressive resistance training [39-43].

Concerning QOL, aerobic exercise associated with greater significant increase in the SF-36 subscale mean values than resisted exercise. Many previous studies approved these findings that indicate that subjects psychological wellbeing and QOL associated with physical exercises [44-47]. Mahendra, reported that aerobic exercise training for 3 months improved SF-36 score of in patients with Alzheimer's disease [48]. However, Bowen et al. proved that postmenopausal women experienced improved QOL following twelve months of exercise intervention [49].In addition, Sørensen, et al. mentioned that 12-month exercise trial improved HRQOL of elderly individuals [50]. Similarly, Imayama, et al. stated that twelve months of moderate to vigorous strength training improved HRQOL improved among overweight men following [51]. In addition, there are evidences reported that exercise training with higher intensity improved mental 
Table 1: Participants baseline and demographic criteria.

\begin{tabular}{|c|c|c|c|}
\hline Characteristic & Group (A) & Group (B) & Significance \\
\hline Age (years) & $63.71 \pm 3.95$ & $64.24 \pm 3.67$ & $p>0.05$ \\
\hline Gender (male/female) & $13 / 12$ & $11 / 14$ & $p>0.05$ \\
\hline Weight $(\mathrm{kg})$ & $66.13 \pm 5.84$ & $64.73 \pm 4.96$ & $p>0.05$ \\
\hline Height $(\mathrm{m})$ & $1.71 \pm 0.08$ & $1.66 \pm 0.07$ & $p>0.05$ \\
\hline BMI $\left(\mathrm{kg} / \mathrm{m}^{2}\right)$ & $22.76 \pm 3.15$ & $23.48 \pm 3.26$ & $p>0.05$ \\
\hline SBP $(\mathrm{mmHg})$ & $135.92 \pm 11.34$ & $139.27 \pm 10.72$ & $p>0.05$ \\
\hline DBP $(\mathrm{mmHg})$ & $82.15 \pm 8.43$ & $84.18 \pm 7.24$ & $p>0.05$ \\
\hline Glucose $(\mathrm{mmol} / \mathrm{L})$ & $5.12 \pm 0.63$ & $5.26 \pm 0.71$ & $p>0.05$ \\
\hline ALT $(\mathrm{U} / \mathrm{L})$ & $38.67 \pm 6.22$ & $40.28 \pm 5.65$ & $p>0.05$ \\
\hline AST $(\mathrm{U} / \mathrm{L})$ & $26.53 \pm 3.85$ & $27.74 \pm 3.92$ & $p>0.05$ \\
\hline $\mathrm{HR}_{\max }(\mathrm{beat} / \mathrm{min})$ & $153.23 \pm 9.67$ & $150.72 \pm 8.95$ & $p>0.05$ \\
\hline
\end{tabular}

BMI: Body Mass Index; SBP: Systolic Blood Pressure; DBP: Diastolic Blood Pressure; AST: Aspartate Aminotransferase; SBP: Systolic Blood Pressure; HR ${ }_{\text {max }}$ : Maximum Heart Rate.

Table 2: Statistical analysis of the immunological parameters before and at the end of the study of group (A).

\begin{tabular}{|c|c|c|c|c|}
\hline & \multicolumn{2}{|c|}{ Mean + SD } & \multirow{2}{*}{$t$ - value } & \multirow{2}{*}{ Significance } \\
\hline & Pre & Post & & \\
\hline CD3 count $\left(10^{9} / \mathrm{L}\right)$ & $1.95 \pm 0.83$ & $1.24 \pm 0.66^{*}$ & 5.37 & $p<0.05$ \\
\hline CD4 count $\left(10^{9} / \mathrm{L}\right)$ & $1.73 \pm 0.94$ & $1.12 \pm 0.72^{*}$ & 5.64 & $p<0.05$ \\
\hline CD8 count $\left(10^{9} / \mathrm{L}\right)$ & $0.97 \pm 0.32$ & $0.51 \pm 0.24^{*}$ & 4.83 & $p<0.05$ \\
\hline CD4/CD8 ratio & $1.69 \pm 0.81$ & $1.14 \pm 0.69^{*}$ & 5.16 & $p<0.05$ \\
\hline
\end{tabular}

Table 3: Statistical analysis of SF-36 subscale scores before and at the end of the studyof group (A).

\begin{tabular}{|c|c|c|c|c|}
\hline \multirow{2}{*}{ SF-36 subscale variables } & \multicolumn{2}{|c|}{ Mean \pm SD } & \multirow{2}{*}{$t$ - value } & \multirow{2}{*}{ Significance } \\
\hline & Before & After & & \\
\hline SF-36: Health transition & $2.73 \pm 0.94$ & $1.53 \pm 0.65^{\star}$ & 4.31 & $p<0.05$ \\
\hline SF-36: Physical functioning & $71.76 \pm 7.23$ & $82.11 \pm 7.83^{*}$ & 6.19 & $p<0.05$ \\
\hline SF-36: Role functioning: Physical & $77.54 \pm 8.36$ & $89.32 \pm 9.27^{*}$ & 7.13 & $p<0.05$ \\
\hline SF-36: Bodily pain & $76.81 \pm 8.19$ & $67.58 \pm 6.73^{*}$ & 6.97 & $p<0.05$ \\
\hline SF-36: General health & $70.65 \pm 7.23$ & $81.87 \pm 7.42^{*}$ & 7.26 & $p<0.05$ \\
\hline SF-36: Vitality & $58.22 \pm 5.15$ & $68.75 \pm 5.91^{*}$ & 5.23 & $p<0.05$ \\
\hline SF-36: Social functioning & $84.37 \pm 7.13$ & $93.41 \pm 7.85^{*}$ & 8.78 & $p<0.05$ \\
\hline SF-36: Role functioning: Emotional & $91.25 \pm 9.84$ & $82.16 \pm 9.57^{*}$ & 8.27 & $p<0.05$ \\
\hline SF-36: Mental health & $83.62 \pm 7.27$ & $71.45 \pm 6.42^{*}$ & 8.11 & $p<0.05$ \\
\hline
\end{tabular}

Table 4: Statistical analysis of the immunological parameters before and at the end of the study of group (B).

\begin{tabular}{|c|c|c|c|c|}
\hline & \multicolumn{2}{|c|}{ Mean + SD } & \multirow{2}{*}{$t$-value } & \multirow{2}{*}{ Significance } \\
\hline & Pre & Post & & \\
\hline CD3 count $\left(10^{9} / \mathrm{L}\right)$ & $1.97 \pm 0.76$ & $1.62 \pm 0.73^{*}$ & 3.21 & $p>0.05$ \\
\hline CD4 count $\left(10^{9} / \mathrm{L}\right)$ & $1.79 \pm 0.84$ & $1.37 \pm 0.82^{*}$ & 2.98 & $p>0.05$ \\
\hline CD8 count $\left(10^{9} / \mathrm{L}\right)$ & $0.98 \pm 0.30$ & $0.76 \pm 0.33^{*}$ & 2.75 & $p>0.05$ \\
\hline CD4/CD8 ratio & $1.72 \pm 0.79$ & $1.51 \pm 0.76^{*}$ & 2.83 & $p>0.05$ \\
\hline
\end{tabular}

Table 5: Statistical analysis of SF-36 subscale scores before and at the end of the study of group (B).

\begin{tabular}{|c|c|c|c|c|}
\hline \multirow{2}{*}{ SF-36 subscale variables } & \multicolumn{2}{|c|}{ Mean + SD } & \multirow{2}{*}{ T-value } & \multirow{2}{*}{ Significance } \\
\hline & Before & After & & \\
\hline SF-36: Health transition & $2.87 \pm 0.81^{*}$ & $2.21 \pm 0.73$ & 2.57 & $p<0.05$ \\
\hline SF-36: Physical functioning & $68.95 \pm 6.74^{*}$ & $75.32 \pm 7.25$ & 3.21 & $p<0.05$ \\
\hline SF-36: Role functioning: Physical & $75.32 \pm 7.56^{*}$ & $82.13 \pm 8.36$ & 3.12 & $p<0.05$ \\
\hline SF-36: Bodily pain & $78.15 \pm 8.23^{*}$ & $73.28 \pm 7.64$ & 3.25 & $p<0.05$ \\
\hline SF-36: General health & $69.72 \pm 7.21^{*}$ & $74.19 \pm 6.32$ & 3.16 & $p<0.05$ \\
\hline SF-36: Vitality & $56.87 \pm 5.92^{*}$ & $61.42 \pm 6.11$ & 3.34 & $p<0.05$ \\
\hline SF-36: Social functioning & $83.95 \pm 8.36^{*}$ & $88.75 \pm 9.13$ & 3.29 & $p<0.05$ \\
\hline SF-36: Role functioning: Emotional & $91.78 \pm 9.81^{*}$ & $86.14 \pm 9.52$ & 3.13 & $p<0.05$ \\
\hline SF-36: Mental health & $83.86 \pm 7.23^{*}$ & $78.23 \pm 6.91$ & 3.37 & $p<0.05$ \\
\hline
\end{tabular}

$\left(^{*}\right)$ indicates a significant difference between the two groups; $p<0.05$.

Table 6: Statistical analysis of the immunological parameters at the end of the study of group (A) and group (B).

\begin{tabular}{|c|c|c|c|c|}
\hline & \multicolumn{2}{|c|}{ Mean + SD } & \multirow{2}{*}{$t$ - value } & \multirow{2}{*}{ Significance } \\
\hline & Group (A) & Group (B) & & \\
\hline CD3 count $\left(10^{9} / \mathrm{L}\right)$ & $1.24 \pm 0.66^{*}$ & $1.62 \pm 0.73$ & 2.98 & $p<0.05$ \\
\hline CD4 count $\left(10^{9} / \mathrm{L}\right)$ & $1.12 \pm 0.72^{*}$ & $1.37 \pm 0.82$ & 2.87 & $p<0.05$ \\
\hline CD8 count $(10 \% / L)$ & $0.51 \pm 0.24^{*}$ & $0.76 \pm 0.33$ & 2.62 & $p<0.05$ \\
\hline CD4/CD8 ratio & $1.14 \pm 0.69^{*}$ & $1.51 \pm 0.76$ & 2.74 & $p<0.05$ \\
\hline
\end{tabular}


Table 7: Statistical analysis of SF-36 subscale scores at the end of the studyof group (A) and group (B).

\begin{tabular}{|c|c|c|c|c|}
\hline \multirow{2}{*}{ SF-36 subscale variables } & \multicolumn{2}{|c|}{ Mean + SD } & \multirow{2}{*}{$t$ - value } & \multirow{2}{*}{ Significance } \\
\hline & Group (A) & Group (B) & & \\
\hline SF-36: Health transition & $1.53 \pm 0.65^{*}$ & $2.21 \pm 0.73$ & 2.62 & $p<0.05$ \\
\hline SF-36: Physical functioning & $82.11 \pm 7.83^{*}$ & $75.32 \pm 7.25$ & 3.31 & $p<0.05$ \\
\hline SF-36: Role functioning: Physical & $89.32 \pm 9.27^{*}$ & $82.13 \pm 8.36$ & 3.43 & $p<0.05$ \\
\hline SF-36: Bodily pain & $67.58 \pm 6.73^{*}$ & $73.28 \pm 7.64$ & 3.22 & $p<0.05$ \\
\hline SF-36: General health & $81.87 \pm 7.42^{*}$ & $74.19 \pm 6.32$ & 3.38 & $p<0.05$ \\
\hline SF-36: Vitality & $68.75 \pm 5.91^{*}$ & $61.42 \pm 6.11$ & 2.76 & $p<0.05$ \\
\hline SF-36: Social functioning & $93.41 \pm 7.85^{\star}$ & $88.75 \pm 9.13$ & 3.47 & $p<0.05$ \\
\hline SF-36: Role functioning: Emotional & $82.16 \pm 9.57^{*}$ & $86.14 \pm 9.52$ & 3.31 & $p<0.05$ \\
\hline SF-36: Mental health & $71.45 \pm 6.42^{*}$ & $78.23 \pm 6.91$ & 3.62 & $p<0.05$ \\
\hline
\end{tabular}

health greater than lower intensity exercise training $[52,53]$. While, Hoffmann, et al. found that AD subjects gained great improvement in QOL and physical fitness following aerobic of moderate intensity [54]. The specific mechanism for improved QOL following exercise training is not well known. However, improved self-perceptions, self-efficacy, activity of neurotransmitters [55-57], neuronal survival [58], synaptic plasticity [61], brain plasticity and vascularization [62-64], along with reduced emotional strain associated with social contact [65].

\section{Conclusion}

Aerobic exercise is more appropriately improve quality of life and immune system among elderly Alzheimer's.

\section{Acknowledgment}

Author wants to express her thanks for staff members of Physical Therapy Department, Faculty of Applied Medical Sciences, King Abdulaziz University for their support in application of therapeutic intervention in this manuscript.

\section{References}

1. Jensen CS, Bahl JM, Østergaard LB, Høgh P, Wermuth L, et al. Exercise as a potential modulator of inflammation in patients with Alzheimer's disease measured in cerebrospinal fluid and plasma. Exp Gerontol. 2019; 121: 91-98.

PubMed: https://www.ncbi.nlm.nih.gov/pubmed/30980923

2. Dos Santos Picanco LC, Ozela PF, de Fatima de Brito Brito M, Pinheiro AA, Padilha EC, et al. Alzheimer's disease: a review from the pathophysiology to diagnosis, new perspectives for pharmacological treatment. Curr Med Chem. 2018; 25: 3141-3159.

PubMed: https://www.ncbi.nlm.nih.gov/pubmed/30191777

3. World Health Organization. Global health and aging. 2019.

4. Rubio-Perez JM, Morillas-Ruiz JM. A review: inflammatory process in Alzheimer's disease, role of cytokines. Scientific World Journal. 2012; 2012: 756357.

PubMed: https://www.ncbi.nlm.nih.gov/pubmed/22566778

5. Melrose RJ, Ettenhofer ML, Harwood D, Achamallah N, Campa O, et al. Cerebral metabolism, cognition and functional abilities in Alzheimer disease. J Geriatr Psychiatry Neurol. 2011; 24: 127-134.

PubMed: https://www.ncbi.nlm.nih.gov/pubmed/21856969

6. Heneka MT, Carson MJ, El Khoury J, Landreth GE, Brosseron F, et al Neuro inflammation in Alzheimer's disease. Lancet Neurol. 2015; 14: 388-405.

PubMed: https://www.ncbi.nlm.nih.gov/pubmed/25792098
7. Heppner FL, Ransohoff RM, Becher B. Immune attack: The role of inflammation in Alzheimer disease. Nat Rev Neurosci. 2015; 16: 358-372. PubMed: https://www.ncbi.nlm.nih.gov/pubmed/25991443

8. Van Eldik LJ, Carrillo MC, Cole PE, Feuerbach D, Greenberg BD, et al. The roles of inflammation and immune mechanisms in Alzheimer's disease. Alzheimers Dement (N Y). 2016; 2: 99-109.

PubMed: https://www.ncbi.nlm.nih.gov/pubmed/29067297

9. Mossaheb N, Zehetmayer S, Jungwirth S, Weissgram S, Rainer M, et al. Are specific symptoms of depression predictive of Alzheimer's dementia? J Clin Psychiatry. 2012; 73: 1009-1015.

PubMed: https://www.ncbi.nlm.nih.gov/pubmed/22687442

10. Lyketsos CG, Carrillo MC, Ryan JM, Khachaturian AS, Trzepacz P, et al. Neuropsychiatric symptoms in Alzheimer's disease. Alzheimers Dement. 2011; 7: 532-539.

PubMed: https://www.ncbi.nlm.nih.gov/pubmed/21889116

11. Heun R, Schoepf D, Potluri R, Natalwala A. Alzheimer's disease and co-morbidity: increased prevalence and possible risk factors of excess mortality in a naturalistic 7-year follow-up. Eur Psychiatry. 2013; 28: 40-48.

PubMed: https://www.ncbi.nlm.nih.gov/pubmed/21924588

12. Alzheimer's Impact Movement. Alzheimer's Disease Caregivers Factsheet. Chicago, IL. Alzheimer's Association; 2017.

13. Idorn M., Hojman P. Exercise-dependent regulation of NK cells in cancer protection. Trends Mol Med. 2016; 22: 565-577.

PubMed: https://www.ncbi.nlm.nih.gov/pubmed/27262760

14. Hayashino $\mathrm{Y}$, Jackson JL, Hirata T, Fukumori N, Nakamura F, et al. Effects of exercise on C-reactive protein, inflammatory cytokine and adipokine in patients with type 2 diabetes: a meta-analysis of randomized controlled trials. Metabol Clin Exp. 2014; 63: 431-440.

15. Neefkes-Zonneveld CR, Bakkum AJ, Bishop NC, Tulder MW, Janssen TW. Effect of long-term physical activity and acute exercise on markers of systemic inflammation in persons with chronic spinal cord injury: a systematic review. Arch Phys Med Rehabil. 2015; 96: 30-42. PubMed: https://www.ncbi.nlm.nih.gov/pubmed/25064781

16. Hammonds TL, Gathright EC, Goldstein CM, Penn MS, Hughes JW. Effects of exercise on c-reactive protein in healthy patients and in patients with heart disease: a meta-analysis. Heart Lung. 2016; 45: 273-282.

PubMed: https://www.ncbi.nlm.nih.gov/pubmed/26916454

17. Meneses-Echavez JF, Correa-Bautista JE, González-Jiménez E, Schmidt RJ, Elkins M, et al. The effect of exercise training on mediators of inflammation in breast cancer survivors: a systematic review with meta-analysis. Cancer Epidemiol Biomarkers Prev. 2016; 25: 1009-1017.

PubMed: https://www.ncbi.nlm.nih.gov/pubmed/27197276

18. Radak Z, Hart N, Sarga L, Koltai E, Atalay M, et al. Exercise plays a 
preventive role against Alzheimer's disease. J Alzheimers Dis. 2010 20: 777-783.

PubMed: https://www.ncbi.nlm.nih.gov/pubmed/20182027

19. Hawley JA, Hargreaves M, Joyner MJ, Zierath JR. Integrative biology of exercise. Cell. 2014; 159: 738-749.

PubMed: https://www.ncbi.nlm.nih.gov/pubmed/25417152

20. De la Rosa A, Olaso-Gonzalez G, Arc-Chagnaud C, Millan F, SalvadorPascual A, et al. Physical exercise in the prevention and treatment of Alzheimer's disease. J Sport Health Sci. 2020; 1-11.

21. Ware J, Kosinski M, Gandek B. SF-36 health survey: manual \& interpretation guide. Lincon, RI: Quality Metric Incorporated. 2000.

22. Robergs RA, Landwehr R. The surprising history of the "HRmax=220age" equation. J Exer Physiol. 2002; 5: 1-10.

23. Ramalho AC, de Lourdes Lima M, Nunes F, Cambuí Z, Barbosa C, et al. The effect of resistance versus aerobic training on metabolic contro in patients with type-1 diabetes mellitus. Diabetes Res Clin Pract. 2006; 72: 271-276

PubMed: https://www.ncbi.nlm.nih.gov/pubmed/16406128

24. Vasefi M, Hudson M, Ghaboolian-Zare E. Diet Associated with Inflammation and Alzheimer's Disease. J Alzheimers Dis Rep. 2019; 3: 299-309.

PubMed: https://www.ncbi.nlm.nih.gov/pubmed/31867568

25. Anshu Agrawal, Janet Baulch, Munjal Acharya, Sudhanshu Agrawal Identification of Peripheral Immune Mechanisms playing a protective role in Alzheimer's Disease progression. J Immunol. 2019; 202: 36.

26. Frederiksen KS, Gjerum L, Waldemar G, Hasselbalch SG. Effects of physical exercise on Alzheimer's disease biomarkers: a systematic review of intervention studies. J Alzheimers Dis. 2018; 61: 359-372. PubMed: https://www.ncbi.nlm.nih.gov/pubmed/29154278

27. Kelly A. Exercise-Induced Modulation of Neuroinflammation in Models of Alzheimer's Disease. Brain Plast. 2018; 4: 81-94.

PubMed: https://www.ncbi.nlm.nih.gov/pubmed/30564548

28. Drela N, Kozdron E, Szczypiorski P. Moderate exercise may attenuate some aspects of immunosenescence. BMC Geriatr. 2004; 4: 8-15. PubMed: https://www.ncbi.nlm.nih.gov/pubmed/15456521

29. Nieman DC, Cook VD, Henson DA, Suttles J, Rejeski WJ, et al. Moderate exercise training and natural killer cell cytotoxic activity in breast cancer patients. Int J Sports Med. 1995; 16: 334-337.

PubMed: https://www.ncbi.nlm.nih.gov/pubmed/7558532

30. Shinkai S, Kohno H, Kimura K, Komura T, Asai $H$, et al. Physical activity and immune senescence in men. Med Sci Sports Exerc. 1995; 27: $1516-1526$

PubMed: https://www.ncbi.nlm.nih.gov/pubmed/8587488

31. Crist DM, Mackinnon LT, Thomson RF, Atterbom HA, Egan PA Physical exercise increases natural cellular-mediated tumor cytotoxicity in the elderly women. Gerontology. 1989; 35: 66-71.

PubMed: https://www.ncbi.nlm.nih.gov/pubmed/2792786

32. Peeri M, Azarbayjani M, Akbarpour M, Ebrahimi M. The effect of aerobic training on the immune system of aging men. Annals Biological Res. 2011; 2: 299-304.

33. Kapasi ZF, Ouslander JG, Schnelle JF, Kutner M, Fahey JL. Effects of an exercise intervention on immunologic parameters in frail elderly nursing home residents. J Gerontol Med Sci. 2003; 58: 636-643. PubMed: https://www.ncbi.nlm.nih.gov/pubmed/12865480

34. Bermon S, Philip P, Candito M, Ferrari P, Dolisic C. Effects of strength exercise and training on the natural killer cell counts in elderly humans. J Sports Med Phys Fitness. 2001; 41: 196-202.

PubMed: https://www.ncbi.nlm.nih.gov/pubmed/11447362
35. Flynn MG, Fahlman M, Brauun WA, Lambert CP, Bouillon LE, et al Effects of resistance training on selected indexes of immune function in elderly women. J Appl Physiol. 1999; 86: 1905-1913. PubMed: https://www.ncbi.nlm.nih.gov/pubmed/10368355

36. Flynn MG, Mcfarlin BK, Philips MD, Stewart LK, Timmerman KL. Tolllike receptor 4 and CD14 mRNA expression are lower in resistive exercise-trained elderly women. J Appl Physiol. 2003; 95: 1833-1842. PubMed: https://www.ncbi.nlm.nih.gov/pubmed/12832426

37. Rall LC, Roubenoff R, Cannon JG, Abad LW, Dinarello CA, et al. Effects of progressive resistance training on immune response in aging and chronic inflammation. Med Sci Sports Exerc. 1996; 28: 1356-1365. PubMed: https://www.ncbi.nlm.nih.gov/pubmed/8933485

38. Mcfarlin BK, Flynn MG, Campbell WW, Stewart LK, Timmerman $\mathrm{KL}$. TLR4 is lower in resistance-trained older women and related to inflammatory cytokines. Med Sci Sports Exerc. 2004; 36: 1876-1883. PubMed: https://www.ncbi.nlm.nih.gov/pubmed/15514501

39. Raso V, Benard G, DA Silva Duarte AJ, Natale VM. Effect of Resistance Training on Immunological Parameters of Healthy Elderly Women. Med Sci Sports Exerc. 2007; 39: 2152-2159.

PubMed: https://www.ncbi.nlm.nih.gov/pubmed/18046186

40. Miles MP, KraemerW J, NindIB C, Grove DS, Leach SK, et al. Strength, workload, anaerobic intensity and the immune response to resistance exercise in women. Acta Physiol Scand. 2003; 178: 155-1563. PubMed: https://www.ncbi.nlm.nih.gov/pubmed/12780390

41. Bruunsgaard $H$, Pedersen BK. Special feature for the olympics: effects of exercise on the immune system in the elderly population. Immunol Cell Biol. 2000; 78: 523-531.

PubMed: https://www.ncbi.nlm.nih.gov/pubmed/11050535

42. Woods JA, Lowder TW, Keylock KT. Can exercise training improve immune function in the aged? Ann N Y Acad Sci. 2002; 959: 117-127. PubMed: https://www.ncbi.nlm.nih.gov/pubmed/11976191

43. Rall LC, Roubenoff R, Cannon JG, Abad LW, Dinarello CA, et al. Effects of progressive resistance training on immune response in aging and chronic inflammation. Med Sci Sport Exerc. 1996; 28: 1356-1365. PubMed: https://www.ncbi.nlm.nih.gov/pubmed/8933485

44. Brown DW, Balluz LS, Heath GW. Associations between recommended levels of physical activity and health-related quality of life-Findings from the 2001 Behavioral Risk Factor Surveillance System (BRFSS) Survey. Prev Med. 2003; 37: 520-528.

PubMed: https://www.ncbi.nlm.nih.gov/pubmed/14572437

45. Segal R, Evans W, Johnson D. Structured exercise improves physical functioning in women with stages I and II breast cancer: results of a randomized controlled trial. J Clin Oncol 2001; 19: 657-665. PubMed: https://www.ncbi.nlm.nih.gov/pubmed/11157015

46. Painter P, Carlson L, Carey S, Paul SM, Myll J. Physical functioning and health-related quality-of-life changes with exercise training in hemodialysis patients. Am J Kidney Dis. 2000; 35: 482-492.

PubMed: https://www.ncbi.nlm.nih.gov/pubmed/10692275

47. Schmitz N, Kruse J, Kugler J. The association between physical exercises and health-related quality of life in subjects with mental disorders: results from a cross-sectional survey. Preventive Medicine 2004; 39: 1200-1207.

PubMed: https://www.ncbi.nlm.nih.gov/pubmed/15539056

48. Mahendra N. Exercise and behavioural management training improves physical health and reduces depression in people with Alzheimer's disease. Evidence-based Healthcare. 2004; 8: 77-79.

49. Bowen DJ, Fesinmeyer MD, Yasui Y, Tworoger S, Ulrich CM, et al Randomized trial of exercise in sedentary middle aged women: effects on quality of life. Int J Behav Nutr Phys Act. 2006; 3: 34.

PubMed: https://www.ncbi.nlm.nih.gov/pubmed/17020620 
50. Sørensen M, Anderssen S, Hjerman I, Holme I, Ursin H. The effect of exercise and diet on mental health and quality of life in middle-aged individuals with elevated risk factors for cardiovascular disease. J Sports Sci. 1999; 17: 369-377.

PubMed: https://www.ncbi.nlm.nih.gov/pubmed/10413264

51. Imayama I, Alfano CM, Cadmus Bertram LA, Wang C, Xiao L, et al. Effects of 12-month exercise on health-related quality of life: a randomized controlled trial. Prev Med. 2011; 52: 344-351.

PubMed: https://www.ncbi.nlm.nih.gov/pubmed/21371498

52. Abu-Omar K, Rütten A, Lehtinen V. Mental health and physical activity in the European Union. Soz Praventivmed. 2004; 49: 301-309. PubMed: https://www.ncbi.nlm.nih.gov/pubmed/15497649

53. McAuley E, Mailey E, Mullen S, Szabo A, Wojcicki T, et al. Growth trajectories of exercise self-efficacy in older adults: influence of measures and initial status. Health Psychol. 2011; 30: 75-83.

PubMed: https://www.ncbi.nlm.nih.gov/pubmed/21038962

54. Hoffmann K, Frederiksen KS, Sobol NA, Beyer N, Vogel A, et al Preserving cognition, quality of life, physical health and functional ability in Alzheimer's disease: the effect of physical exercise (ADEX trial): rationale and design. Neuroepidemiology. 2013; 41: 198-207 . PubMed: https://www.ncbi.nlm.nih.gov/pubmed/24135720

55. Paluska SA, Schwenk TL. Physical activity and mental health: current concepts. Sports Med. 2000; 29: 167- 180.

PubMed: https://www.ncbi.nlm.nih.gov/pubmed/10739267

56. Nichol K, Deeny SP, Seif J, Camaclang K, Cotman CW. Exercise improves cognition and hippocampal plasticity in APOE epsilon4 mice. Alzheimers Dement. 2009; 5: 287-294.

PubMed: https://www.ncbi.nlm.nih.gov/pubmed/19560099

57. van Praag H, Christie BR, Sejnowski TJ, Gage FH. Running enhances neurogenesis, learning, and long-term potentiation in mice. Proc Natl Acad Sci U S A. 1999; 96: 13427-13431.

PubMed: https://www.ncbi.nlm.nih.gov/pmc/articles/PMC23964/
58. Barde YA. Neurotrophins: a family of proteins supporting the survival of neurons. Prog Clin Biol Res. 1994; 390: 45-56.

PubMed: https://www.ncbi.nlm.nih.gov/pubmed/7724649

59. Stummer W, Weber K, Tranmer B, Baethmann A, Kempski O. Reduced mortality and brain damage after locomotor activity in gerbil forebrain ischemia. Stroke. 1994; 25: 1862-1869.

PubMed: https://www.ncbi.nlm.nih.gov/pubmed/8073470

60. Carro E, Trejo JL, Busiguina S, Torres-Aleman I. Circulating insulinlike growth factor I mediates the protective effects of physical exercise against brain insults of different etiology and anatomy. J Neurosci. 2001; 21: 5678-5684.

PubMed: https://www.ncbi.nlm.nih.gov/pubmed/11466439

61. Lu B, Chow A. Neurotrophins and hippocampal synaptic transmission and plasticity. J Neurosci Res. 1999; 58: 76-87.

PubMed: https://www.ncbi.nlm.nih.gov/pubmed/10491573

62. Black JE, Isaacs KR, Anderson BJ, Alcantara AA, Greenough WT. Learning causes synaptogenesis, whereas motor activity causes angiogenesis, in cerebellar cortex of adult rats. Proc Natl Acad Sci USA. 1990; 87: 5568-5572.

PubMed: https://www.ncbi.nlm.nih.gov/pubmed/1695380

63. Isaacs KR, Anderson BJ, Alcantara AA, Black JE, Greenough WT. Exercise and the brain: angiogenesis in the adult rat cerebellum after vigorous physical activity and motor skill learning. J Cereb Blood Flow Metab. 1992; 12: 110-119.

PubMed: https://www.ncbi.nlm.nih.gov/pubmed/1370068

64. Cotman CW, Berchtold NC. Exercise: a behavioral intervention to enhance brain health and plasticity. Trends Neurosci. 2002; 25: 295-301. PubMed: https://www.ncbi.nlm.nih.gov/pubmed/12086747

65. Greist JH, Klein MH, Eischens RJ, Faris J, Gurman AS, et al. Running as treatment for depression. Compr Psychiatry. 1979; 20: 41-54. PubMed: https://www.ncbi.nlm.nih.gov/pubmed/759100 